\title{
The effectiveness of cooling conditions on temperature of canine EDTA whole blood samples
}

\author{
Karen M Tobias ${ }^{\text {Corresp., }}{ }^{1}$, Leslie Serrano ${ }^{2}{ }^{\text {, Xiaocun Sun }}{ }^{3}$, Bente Flatland ${ }^{4}$ \\ ${ }^{1}$ Department of Small Animal Clinical Sciences, College of Veterinary Medicine, University of Tennessee, Knoxville, TN, US \\ 2 College of Veterinary Medicine, University of Tennessee - Knoxville, Knoxville, Tennessee, United States \\ 3 Office of Information Technology, University of Tennessee - Knoxville, Knoxville, Tennessee, United States \\ 4 Department of Biomedical and Diagnostic Sciences, University of Tennessee - Knoxville, Knoxville, Tennessee, United States \\ Corresponding Author: Karen M Tobias \\ Email address: ktobias@utk.edu
}

Background. Preanalytic factors such as time and temperature can have significant effects on laboratory test results. For example, ammonium concentration will increase $31 \%$ in blood samples stored at room temperature for 30 minutes before centrifugation. To reduce preanalytic error, blood samples may be placed in precooled tubes and chilled on ice or in ice water baths; however, the effectiveness of these modalities in cooling blood samples has not been formally evaluated. The purpose of this study was to evaluate the effectiveness of various cooling modalities on reducing temperature of EDTA whole blood samples.

Methods. Pooled samples of canine EDTA whole blood were divided into two aliquots. Saline was added to one aliquot to produce a packed cell volume (PCV) of $40 \%$ and to the second aliquot to produce a PCV of $20 \%$ (simulated anemia). Thirty samples from each aliquot were warmed to $37.7^{\circ} \mathrm{C}$ and cooled in $2 \mathrm{ml}$ allotments under one of three conditions: in ice, in ice after transfer to a precooled tube, or in an ice water bath. Temperature of each sample was recorded at one minute intervals for 15 minutes.

Results. Within treatment conditions, sample PCV had no significant effect on cooling. Cooling in ice water was significantly faster than cooling in ice only or transferring the sample to a precooled tube and cooling it on ice. Mean temperature of samples cooled in ice water was significantly lower at 15 minutes than mean temperatures of those cooled in ice, whether or not the tube was precooled. By 4 minutes, samples cooled in an ice water bath had reached mean temperatures less than $4{ }^{\circ} \mathrm{C}$ (refrigeration temperature), while samples cooled in other conditions remained above $4.0^{\circ} \mathrm{C}$ for at least 11 minutes. For samples with a PCV of $40 \%$, precooling the tube had no significant effect on rate of cooling on ice. For samples with a PCV of $20 \%$, transfer to a precooled tube resulted in a significantly faster rate of cooling than direct placement of the warmed tube onto ice.

Discussion. Canine EDTA whole blood samples cool most rapidly and to a greater degree when placed in an ice-water bath rather than in ice. Samples stored on ice water can rapidly drop below normal refrigeration temperatures; this should be taken into consideration when using this cooling modality. 
1 The effectiveness of cooling conditions on temperature of canine EDTA whole blood samples

2 Karen M. Tobias ${ }^{1}$, Leslie Serrano ${ }^{1}$, Xiaocun Sun $^{2}$, and Bente Flatland ${ }^{3}$

3

$4 \quad{ }^{1}$ Departments of Small Animal Clinical Sciences, College of Veterinary Medicine, University of Tennessee

5 Institute of Agriculture, Knoxville TN, USA

$6{ }^{2}$ Office of Information Technology (Sun) at the University of Tennessee; Knoxville TN, USA.

$7 \quad{ }^{3}$ Department of Biomedical and Diagnostic Sciences, College of Veterinary Medicine, University of

8 Tennessee Institute of Agriculture, Knoxville TN, USA

9

10 Corresponding Author: Karen Tobias

11 Email address: ktobias@utk.edu

12 
13 The effectiveness of cooling conditions on temperature of EDTA whole canine blood samples

14

15

16

17

18

19

20

21

22

23

24

25

26

27

28

29

30

31

32

33

34

35

36

37

38

39

40

41

42

43

44

45

46

47

48

\section{Abstract}

Background. Preanalytic factors such as time and temperature can have significant effects on laboratory test results. For example, ammonium concentration will increase $31 \%$ in blood samples stored at room temperature for 30 minutes before centrifugation. To reduce preanalytic error, blood samples may be placed in precooled tubes and chilled on ice or in ice water baths; however, the effectiveness of these modalities in cooling blood samples has not been formally evaluated. The purpose of this study was to evaluate the effectiveness of various cooling modalities on reducing temperature of EDTA whole blood samples.

Methods. Pooled samples of canine EDTA whole blood were divided into two aliquots. Saline was added to one aliquot to produce a packed cell volume (PCV) of $40 \%$ and to the second aliquot to produce a PCV of $20 \%$ (simulated anemia). Thirty samples from each aliquot were warmed to $37.7^{\circ} \mathrm{C}$ and cooled in $2 \mathrm{ml}$ allotments under one of three conditions: in ice, in ice after transfer to a precooled tube, or in an ice water bath. Temperature of each sample was recorded at one minute intervals for 15 minutes.

Results. Within treatment conditions, sample PCV had no significant effect on cooling. Cooling in ice water was significantly faster than cooling in ice only or transferring the sample to a precooled tube and cooling it on ice. Mean temperature of samples cooled in ice water was significantly lower at 15 minutes than mean temperatures of those cooled in ice, whether or not the tube was precooled. By 4 minutes, samples cooled in an ice water bath had reached mean temperatures less than $4^{\circ} \mathrm{C}$ (refrigeration temperature), while samples cooled in other conditions remained above $4.0^{\circ} \mathrm{C}$ for at least 11 minutes. For samples with a PCV of $40 \%$, precooling the tube had no significant effect on rate of cooling on ice. For samples with a PCV of $20 \%$, transfer to a precooled tube resulted in a significantly faster rate of cooling than direct placement of the warmed tube onto ice.

Discussion. Canine EDTA whole blood samples cool most rapidly and to a greater degree when placed in an ice-water bath rather than in ice. Samples stored on ice water can rapidly drop below normal refrigeration temperatures; this should be taken into consideration when using this cooling modality.

2 


\section{Introduction}

Laboratory error can occur in the pre-, intra-, and postanalytic phases of sample analysis, with $31.6 \%$ to $75 \%$ of errors occurring in the preanalytic phase. ${ }^{1}$ Examples of preanalytic errors include lack of patient fasting, iatrogenic hemolysis, mislabeling of samples, and inappropriate sample storage. Measurement of whole blood and plasma ammonium concentration is useful for diagnosis of portovascular anomalies and hepatic encephalopathy and for monitoring response to treatment in people and dogs. ${ }^{2-4}$ Ammonium is very labile, and time and temperature have significant preanalytic effects on laboratory results in people. ${ }^{5,6}$ For example, storage of whole blood at 4 degrees $\mathrm{C}$ and 22 degrees $\mathrm{C}$ for 1 hour results in increases in plasma ammonium of $31.7 \%$ and $39.9 \%$, respectively. ${ }^{6}$ Heparinized whole blood samples stored in ice water for 30 minutes before centrifugation have significantly less increases in plasma ammonium than those stored at room temperature for 30 minutes before centrifugation (13\% vs. $31 \%$, respectively). ${ }^{7}$ Recommendations for handling samples for ammonium measurement include collecting the blood in a stoppered ammonia-free vacuum tube, placing the tube immediately in an ice or ice water bath, and separating the plasma from the sample within 15 minutes of collection to prevent spontaneous ammonium generation. ${ }^{2,7-10}$ Some authors also recommend using a precooled vacuum sample tube to speed the specimen cooling process. ${ }^{2,10}$ Effectiveness of precooling tubes and of use of ice versus ice water baths for cooling blood samples has not been formally evaluated and reported in the medical literature. The purpose of this study was to compare different sample cooling protocols to determine which would be most effective for rapidly reducing blood sample temperature before centrifugation and analysis. This work is part of an effort to optimize sample handling protocols for ammonium analysis in our hospital.

\section{Materials and Methods}

Leftover refrigerated canine EDTA whole blood samples were obtained from the University of Tennessee College of Veterinary Medicine Clinical Pathology Laboratory and used for testing. All samples were 7 days old at the time of entry into the study and had been stored at $4{ }^{\circ} \mathrm{C}$. Samples were pooled, and a packed cell volume (PCV) of the resulting pool was measured. The pool was then divided into two aliquots. For aliquot 1 (normal PCV), physiologic saline was added to decrease the PCV to $40 \%$. For aliquot 2 (simulated anemia), physiologic saline was added to decrease the PCV to $20 \%$. Each aliquot was divided into $2 \mathrm{ml}$ samples and $2.5 \mathrm{ml}$ samples that were placed in a $13 \times 75 \mathrm{~mm}$, stoppered, EDTA-free sample tubes (Vacutainer ${ }^{\circledR}$, Becton Dickinson Company) and kept refrigerated at $4{ }^{\circ} \mathrm{C}$ until testing. In all test conditions, the cooling rate of $2 \mathrm{mls}$ of blood was to be evaluated; however, placement of a warmed blood sample into a precooled tube required transfer of the blood. For those samples, an extra $0.5 \mathrm{ml}$ of blood was included in the storage tubes to ensure a full $2 \mathrm{mls}$ of blood was transferred at the time of testing. Two mls of blood was chosen because it is the standard volume collected in a $13 \times 75$ mm containing $3.6 \mathrm{mg}$ of potassium EDTA; additionally, that volume of blood forms a column less than $30 \mathrm{~mm}$ in height within the tube, ensuring that the surface level of the blood will rest several $\mathrm{cm}$ below the surface level of the ice or ice/water.

The testing apparatus consisted of a lidded $148 \mathrm{ml}$ plastic specimen cup (Fisherbrand ${ }^{\mathrm{TM}}$ multipurpose specimen storage containers, Fisher Scientific), a temperature probe with an 
90

91

92

93

94

95

96

97

98

99

100

101

102

103

104

105

106

107

108

109

110

111

112

113

114

115

116

117

118

119

120

121

122

123

124

125

126

127

128

129

130

131

accuracy of $\pm 0.3^{\circ} \mathrm{C}$ (Fisher Scientific ${ }^{\mathrm{TM}}$ Traceable $^{\mathrm{TM}}$ Hi-Accuracy Thermometer), and a support stand with clamps. A hole the diameter of the sample tube was drilled in the lid of the specimen cup to allow the tube to be inserted through the lid and to be held upright and centered in the specimen cup with the bottom of the tube touching the bottom of the cup and the stopper resting above the surface of the lid. A hole was drilled in a tube stopper, which was secured onto the temperature probe and used for all measurements, so that the temperature probe would maintain the same location and depth in each tube and so that each tube remained stoppered for the entirety of the test.

Immediately before testing, stoppered blood tubes were placed on a rack in a $38^{\circ} \mathrm{C}$ water bath and warmed to $37.7^{\circ} \mathrm{C}$. Samples from each aliquot (normal PCV or anemic PCV) were tested under one of three cooling conditions: 1, Ice bath; 2, Precooled $(\mathrm{P} / \mathrm{C})$ tube in ice bath; and 3, Ice water bath. For Condition 1 (ice bath), the specimen cup was filled with $70 \mathrm{gm}$ of crushed ice and capped with the prefabricated lid. A $13 \times 75 \mathrm{~mm}$ stoppered sample tube containing $2 \mathrm{mls}$ of warmed $\left(37.7^{\circ} \mathrm{C}\right)$ blood was inserted through the hole in the lid, and its stopper was replaced with the stopper-temperature probe unit. For Condition 2 (P/C tube in ice bath), an empty $13 \times 75$ $\mathrm{mm}$ stoppered sample tube was placed on ice. After 5 minutes, $2 \mathrm{mls}$ of blood were withdrawn from a storage tube containing $2.5 \mathrm{mls}$ of warmed $\left(37.7^{\circ} \mathrm{C}\right)$ blood and transferred into the $\mathrm{P} / \mathrm{C}$ sample tube, which was then positioned in a cup containing $70 \mathrm{~g}$ of crushed ice and capped with the stopper-temperature probe unit, as for Condition 1. For Condition 3 (Ice water bath), the specimen cup was filled with $70 \mathrm{~g}$ of crushed ice and $45 \mathrm{~g}$ of water and covered with the prefabricated lid. Immediately thereafter, a $13 \times 75 \mathrm{~mm}$ sample tube containing $2 \mathrm{mls}$ of warmed blood was capped and positioned in the specimen cup, as described for Conditions 1 and 2. In each condition, the surface of the blood sample rested several $\mathrm{cm}$ below the surface of the cooling agent (ice or ice water). Temperature of each sample was recorded at one-minute intervals for 15 minutes.

Six treatments (ice bath/PCV 20\%; ice bath/PCV 40\%; $\mathrm{P} / \mathrm{C}$ in ice/PCV 20\%; $\mathrm{P} / \mathrm{C}$ in ice/PCV $40 \%$; ice water/PCV 20\%; ice water/PCV 40\%) were evaluated over 16 time points (0 to 15 minutes), with 30 replicates per treatment. Temperature data were evaluated using a one-way ANOVA with repeated measures, with treatment as the between subject factor and time as the within subject factor. Time was analyzed as a categorical variable and a numeric variable, respectively, to estimate the effect of treatment for each time interval and the overall trend of time effect. The rate of cooling (speed) data was analyzed using one-way ANOVA. Rate of cooling was computed using the range of the temperature change divided by the total time interval (15 minutes). Ranked transformation was applied when data violated ANOVA assumptions such as non-normality and unequal variance. Post hoc multiple comparison was conducted with Tukey's adjust. To analyze the trend of time, time was included in the model as a numeric factor whereas treatment as a categorical variable. Significance was set at $\mathrm{p}<0.05$ and all analysis was processed in SAS9.4 TS1M1 for Windows x64 (Cary, NC).

\section{Results}

Results are summarized in Tables 1 and 2. Time exhibited a significant decreasing effect $(p<0.0001)$ on temperature when controlling for the treatment effect. However, time and 
132 treatment interaction effect was also significant $(\mathrm{p}<0.0001)$, indicating the effect of time was

133

134

135

136

137

138

139

140

141

142

143

144

145

146

147

148

149

150

151

152

153

154

155

156

157

158

159

160

161

162

163

164

165

166

167

168

169

170

171

172

173

174

175

176

dependent on treatment.

Within treatment conditions (ice, ice water, and P/C tube on ice), sample PCV had no significant effect on cooling. Cooling in ice water was significantly faster than cooling on ice only or P/C tube on ice (Figure 1). Regardless of PCV, mean temperatures of samples cooled in ice water were significantly lower at 15 minutes than mean temperatures of samples cooled on ice only or in $\mathrm{P} / \mathrm{C}$ tube on ice $(\mathrm{p}<0.0001)$. By 4 minutes, samples cooled in ice water had reached mean temperatures less than $4^{\circ} \mathrm{C}$ (that of refrigeration), regardless of PCV.

For samples with a $20 \% \mathrm{PCV}$, mean temperature at 4 minutes of cooling in ice water was significantly lower than mean temperature at 14 minutes for samples cooled on ice only $(\mathrm{p}=0.0148)$ or at 11 minutes for samples in $\mathrm{P} / \mathrm{C}$ tube on ice $(\mathrm{p}<0.0001)$. Of samples with a $40 \%$ $\mathrm{PCV}$, mean temperature at 4 minutes of samples cooled in ice water was significantly cooler than mean temperature at 13 minutes for samples cooled on ice only $(\mathrm{p}=0.0335)$ or at 12 minutes for samples in $\mathrm{P} / \mathrm{C}$ tube on ice $(\mathrm{p}<0.0001)$.

For samples with a PCV of $40 \%$, there was no difference in mean temperatures or rate of cooling of samples placed on ice and those placed in $\mathrm{P} / \mathrm{C}$ tubes on ice. For samples with a $20 \%$ packed cell volume, however, rate of cooling was significantly faster for samples in a $\mathrm{P} / \mathrm{C}$ tube on ice, versus those placed on ice. From 4 to 13 minutes, mean temperatures of samples with a $20 \%$ $\mathrm{PCV}$ that were placed in a $\mathrm{P} / \mathrm{C}$ tube on ice were significantly lower than mean temperatures of samples placed on ice without precooling the tube.

\section{Discussion}

In this study we determined blood sample cooling occurred more rapidly and to a greater extent when samples were placed in an ice water bath versus in a cup of crushed ice. With a closed system that lacks motion, as described in this study, cooling of blood samples relies primarily on conduction. Conduction is the flow of heat that reduces the temperature difference between two materials. ${ }^{11}$ Transfer of heat via conduction relies on exchange of kinetic energy between microscopic particles within substances: in this case, between the blood and the tube containing it, and between the tube and its external environment (ice or ice water). Variables important to heat transfer include the material's innate ability to conduct heat (thermal conductivity), the thickness of the materials involved, the surface area available for heat transfer, and the temperature difference across these surfaces. ${ }^{11.12}$ In our study, sample volume and tube size and thickness were standardized; therefore, differences in rates of cooling were primarily dependent on the thermal conductivity of the materials used for cooling, the amount of contact each of these substances has with the tube, and the temperature of the cooling condition. Rate of cooling may change if the tube contains more or less blood or if the surface level of the blood is not totally submerged below the surface level of the ice or ice water.

In our study, cooling was performed using closed systems containing crushed ice or ice water. Ice water consists of two major components, ice and water, with thermal conductivity coefficients of $2.1 \mathrm{~W} /(\mathrm{m} \mathrm{K})$ at $0^{\circ} \mathrm{C}$ for ice and $0.6 \mathrm{~W} /(\mathrm{m} \mathrm{K})$ at $25^{\circ} \mathrm{C}$ for water. Crushed ice 
177 contains a small amount of liquid water but primarily consists of ice and air, the latter of which 178 has a thermal conductivity coefficient of $0.024 \mathrm{~W} /(\mathrm{m} \mathrm{K})$ at $0^{\circ} \mathrm{C}$. Because thermal conductivity of 179 water is much greater than air, it serves as a better conductor of heat and thus increases the rate 180 of heat loss from the sample. ${ }^{12}$

181

182

183

184

185

186

187

188

189

190

191

192

193

194

195

196

197

198

199

200

201

202

203

204

205

206

207

208

209

210

211

212

213

214

215

216

217

218

219

220

221

222

Surface areas of the cooling materials used in this study cannot be calculated, since pieces of crushed ice varied greatly in size and shape and thus in their ability to contact the surface of the tube. Therefore, a variable amount of air was present within the crushed ice condition. Addition of water to the ice increased the surface area for diffusion of heat via a material with higher conductivity, thereby speeding heat transfer. Temperature of the condition (ice or ice water) was not measured in this experiment. Temperature difference was likely greater between the sample and the ice water, however, since pockets of air within the crushed ice could have maintained higher temperatures than the ice water.

A PCV of $40 \%$ was chosen as the normal packed cell volume in this study because it was consistent with the mean PCV $(39.6 \% \pm 6.4)$ of 102 dogs presenting for ammonium measurement at our clinic (unpublished data). The samples were diluted with physiologic saline (versus plasma) to maintain consistency, since plasma components can vary among dogs.

Between conditions, packed cell volume had no significant effect on rates of cooling. This is not surprising, since the coefficients of thermal conductivity for EDTA blood and EDTA plasma are approximately the same as that of water. ${ }^{13}$ However, while no significant difference was noted between cooling on ice and use of a precooled tube plus cooling on ice for samples with a PCV of $40 \%$, use of a precooled tube produced a significantly greater reduction in temperature for samples with a PCV of $20 \%$. Rather than being an effect of PCV per se, a possible cause for this finding is an effect of tube handling. To mimic clinic conditions used in sample collection for ammonium analysis, precooling was performed by placement of a stoppered tube on ice. Leaving the stopper in place during sample collection and transport prevents inadvertent contamination of the tube with environmental factors, such as cigarette smoke or cleaning agents, that can result in erroneously increases in measured ammonium. ${ }^{14}$ However, in our system the stoppered tubes were manually removed from the ice bath to have their caps removed, which could have increased temperature of the tube by introduction of warmer environmental air and by heat transfer from the hand to the tube surface. Although it is unlikely, we cannot rule out that tubes containing blood with a 40\% PCV were handled more or kept out of the ice bath longer during cap removal and tube filling, resulting in a smaller temperature difference between the sample and the tube and a slower rate of cooling.

One concern with use of an ice water bath for cooling of diagnostic whole blood specimens is whether the rapid rate of cooling and attainment of "near-freezing" temperatures will adversely affect samples. In terms of ammonium concentration, sample storage at $0^{\circ} \mathrm{C}$ to $-80^{\circ} \mathrm{C}$ will reduce, but not halt, spontaneous ammonia generation. ${ }^{6,15,16}$ Both rapid increases in temperature and intracellular formation of ice crystals can increase red cell fragility, which can result in hemolysis and increased plasma ammonium concentrations. ${ }^{7,10,17,18}$ In one study, rate of ammonia increase in whole blood significantly correlated with erythrocyte count and plasma alanine aminotransferase and gamma glutamyltransferase concentrations when blood was stored at $20^{\circ} \mathrm{C}$ but not when stored at $0^{\circ} \mathrm{C}$. ${ }^{16}$ Although hemolysis was not specifically investigated in that study, those findings argue that RBC-related increases in ammonium concentration may be

PeerJ reviewing PDF | (2016:08:12929:2:0:NEW 22 Oct 2016) 
223

224

225

226

227

228

229

230

231

232

233

234

235

236

237

238

239

240

241

242

243

244

245

246

247

248

249

250

251

252

253

254

255

256

257

258

259

260

related to cell metabolism rather than hemolysis. Hemolysis was not evaluated in our study, and further study of whether rapid specimen cooling in an ice water bath induces hemolysis, and to what magnitude, is warranted.

\section{Conclusions}

Canine EDTA whole blood samples cool most rapidly and to a greater degree when placed in an ice water bath rather than in ice. Use of ice water baths may be important for reducing preanalytical error in the measurement of ammonium and other analytes altered by metabolic processes that continue within blood samples after they are collected; further research is needed in this area. Samples stored on ice water can rapidly drop below normal refrigeration temperatures; this should be taken into consideration when using this cooling modality.

\section{References}

1. Bonini P, Plebani M, Ceriotti F, Rubbgoli F: Errors in laboratory medicine. Clin Chem 2002;48:691-698.

2. Barsotti RJ: Measurement of ammonia in blood. J Pediatr 2001;138:S11-S20.

3. Lidbury JA, Ivanek R, Suchodolski JS, Steiner JM: Putative precipitating factors for hepatic encephalopathy in dogs: 118 cases. J Am Vet Med Assoc 2015;247:176-183.

4. Natesan V, Mani R, Arumugam R: Clinical aspects of urea cycle dysfunction and altered brain energy metabolism on modulation of glutamate receptors and transporters in acute and chronic hyperammonemia. Biomed Pharmacother 2016;81:192-202.

5. Heins M, Heil W, Withold W: Storage of serum or whole blood samples? Effects of time and temperature on 22 serum analytes. Eur J Clin Chem Clin Biochem 1995;33:231-238

6. Howanitz JH, Howanitz PJ, Skrodski CA, Iwanski JA: Influences of specimen processing and storage conditions on results for plasma ammonia. Clin Chem 1984;30:906-908.

7. Nikolac N, Omazic J, Simundic AM: The evidence based practice for optimal sample quality of ammonia measurement. Clin Biochem 2014;47:991-995.

8. Association for Clinical Biochemistry 2012: Ammonia (plasma, blood). www.acb.org.uk/Nat Lab Med Hbk/Ammonia.pdf Downloaded 8/8/2016

9. Dukic L, Simundic AM: Short-term and long-term storage stability of heparin plasma ammonia. J Clin Pathol 2015;68:288-291.

10. Maranda B, Cousineau J, Allard P, Lambert M. False positives in plasma ammonia measurement and their clinical impact in a pediatric population. Clin Biochem 2007;40:531-535.

11. Radi, HA., Rasmussen, John O: Principles of Physics: For Scientists and Engineers. Springer-Verlag, Berlin Heidelberg, 2013; pp 357-425. 
261 12. Vlachopoulos J, Strutt D: Basic heat transfer and some applications in polymer processing.

262 Plastics Technician's Toolbox 2 (2002): 21-33.

263 13. Ponder E: the coefficient of thermal conductivity of blood and of various tissues. J Gen

264 Physiol 1962,45:545-551.

265 14. Hashim IA, Cuthbert JA: Elevated ammonia concentrations: Potential for pre-analytical and 266 analytical contributing factors. Clin Biochem 2014;47:233-236.

267 15. Da Fonseca-Wolheim F: Deamidation of glutamine by increased plasma gamma

268 glutamyltransferase is a source of rapid ammonia formation in blood and plasma specimens. Clin 269 Chem 1990;36:1479-1482.

270

271 16. Da Fonseca-Wolhelm F: Preanalytical increase of ammonia in blood specimens from

272 healthy subjects. Clin Chem 1990;36(8):1483-1487.

273

274 17. El-Khoury JM, Bunch DR, Wang S: Is the effect of hemolysis on plasma ammonia 275 measurement overrated? Arch Pathol Lab med 2012;136:471-472.

276

277

18. Patterson J, Rousseau A, Kessler RJ, Giger U: In vitro lysis and acute transfusion reactions 278 with hemolysis caused by inappropriate storage of canine red blood cell products. J Vet Intern

279 Med 2011;25:927-933. 
282 Table 1. Summary of treatment comparisons (treatment 1 vs. treatment 2 ) showing effectiveness 283 of blood sample cooling conditions on the mean temperature drop over 15 minutes.

284

\begin{tabular}{|l|l|l|l|l|l|l|}
\hline & $\begin{array}{l}\text { Ice 20\% } \\
\text { PCV }\end{array}$ & $\begin{array}{l}\text { Ice 40\% } \\
\text { PCV }\end{array}$ & $\begin{array}{l}\text { P/C 20\% } \\
\text { PCV }\end{array}$ & $\begin{array}{l}\text { P/C 40\% } \\
\text { PCV }\end{array}$ & $\begin{array}{l}\text { Water/Ice } \\
20 \% \text { PCV }\end{array}$ & $\begin{array}{l}\text { Water/Ice } \\
40 \% \text { PCV }\end{array}$ \\
\hline Ice 20\% PCV & ------ & $\mathrm{p}=0.6405$ & $\mathrm{p}<0.0001$ & ----- & $\mathrm{p}<0.0001$ & ------- \\
\hline Ice 40\% PCV & $\mathrm{p}=0.6405$ & ----- & ----- & $\mathrm{p}=0.7974$ & ----- & $\mathrm{p}<0.0001$ \\
\hline P/C 20\% PCV & $\mathrm{p}<0.0001$ & ----- & ---- & $\mathrm{p}=0.1697$ & $\mathrm{p}<0.0001$ & ----- \\
\hline P/C 40\% PCV & ----- & $\mathrm{p}=0.7974$ & $\mathrm{p}=0.1697$ & ----- & ----- & $\mathrm{p}<0.0001$ \\
\hline $\begin{array}{l}\text { Water/Ice } \\
20 \% \text { PCV }\end{array}$ & $\mathrm{p}<0.0001$ & ----- & $\mathrm{p}<0.0001$ & ----- & ----- & $\mathrm{p}=0.9983$ \\
\hline $\begin{array}{l}\text { Water/Ice } \\
40 \% \text { PCV }\end{array}$ & ----- & $\mathrm{p}<0.0001$ & ----- & $\mathrm{p}<0.0001$ & $\mathrm{p}=0.9983$ & ----- \\
\hline
\end{tabular}

285 Ice = ice bath; $\mathrm{P} / \mathrm{C}=$ precooled tube in ice bath; water/ice = ice water bath; $\mathrm{PCV}=$ packed cell 286 volume; $p$ value $=$ Tukey's adjusted probability value, with significance set at $\mathrm{p}<0.05$.

287 Table 2: Mean temperature $\left({ }^{\circ} \mathrm{C}\right)$ per minute of normal and simulated anemic blood samples for 3 288 cooling conditions.

289

\begin{tabular}{|c|c|c|c|c|c|c|}
\hline $\begin{array}{l}\text { TIME } \\
\text { (MIN) }\end{array}$ & $\begin{array}{l}\text { ICE } \\
20 \% \text { PCV }\end{array}$ & $\begin{array}{l}\text { ICE } \\
40 \% \text { PCV }\end{array}$ & $\begin{array}{l}\text { PRECOOLED } \\
20 \% \text { PCV }\end{array}$ & $\begin{array}{l}\text { PRECOOLED } \\
40 \% \mathrm{PCV}\end{array}$ & $\begin{array}{l}\text { WATER/ICE } \\
20 \% \mathrm{PCV}\end{array}$ & $\begin{array}{l}\text { WATER/ICE } \\
40 \% \mathrm{PCV}\end{array}$ \\
\hline 0 & 37.7 & 37.7 & 37.7 & 37.7 & 37.7 & 37.7 \\
\hline 1 & 27.74 & 27.6 & 22.57 & 23.91 & 17.53 & 19.07 \\
\hline 2 & 21.94 & 21.88 & 17.9 & 19.74 & 9.26 & 10.09 \\
\hline 3 & 18.35 & 17.93 & 14.68 & 16.41 & 5.26 & 5.53 \\
\hline 4 & 15.5 & 15.07 & 12.23 & 13.76 & 3.07 & 3.09 \\
\hline 5 & 13.22 & 12.75 & 10.36 & 11.68 & 1.84 & 1.78 \\
\hline 6 & 11.4 & 10.85 & 8.86 & 9.99 & 1.12 & 1.06 \\
\hline 7 & 9.88 & 9.27 & 7.65 & 8.64 & 0.71 & 0.66 \\
\hline 8 & 8.62 & 7.99 & 6.63 & 7.51 & 0.47 & 0.44 \\
\hline 9 & 7.54 & 6.94 & 5.78 & 6.56 & 0.33 & 0.31 \\
\hline 10 & 6.65 & 6.05 & 5.06 & 5.74 & 0.24 & 0.24 \\
\hline 11 & 5.88 & 5.3 & 4.46 & 5.05 & 0.20 & 0.2 \\
\hline 12 & 5.22 & 4.67 & 3.98 & 4.45 & 0.17 & 0.18 \\
\hline 13 & 4.65 & 4.12 & 3.5 & 3.94 & 0.16 & 0.16 \\
\hline 14 & 4.14 & 3.66 & 3.12 & 3.51 & 0.15 & 0.16 \\
\hline 15 & 3.69 & 3.27 & 2.8 & 3.14 & 0.14 & 0.15 \\
\hline
\end{tabular}

$290 \quad \mathrm{PCV}=$ Packed cell volume; $\mathrm{MIN}=$ minutes

291

292 
293 Figure 1. Mean speed of temperature drop over 15 mins ( \pm SEM) under conditions of ice20\%, 294 ice 40\%, P/C 20\%, P/C\% 40\%, water/ice 20\% and water/ice 40\%(N=30 for each treatment 295 group).

296

297

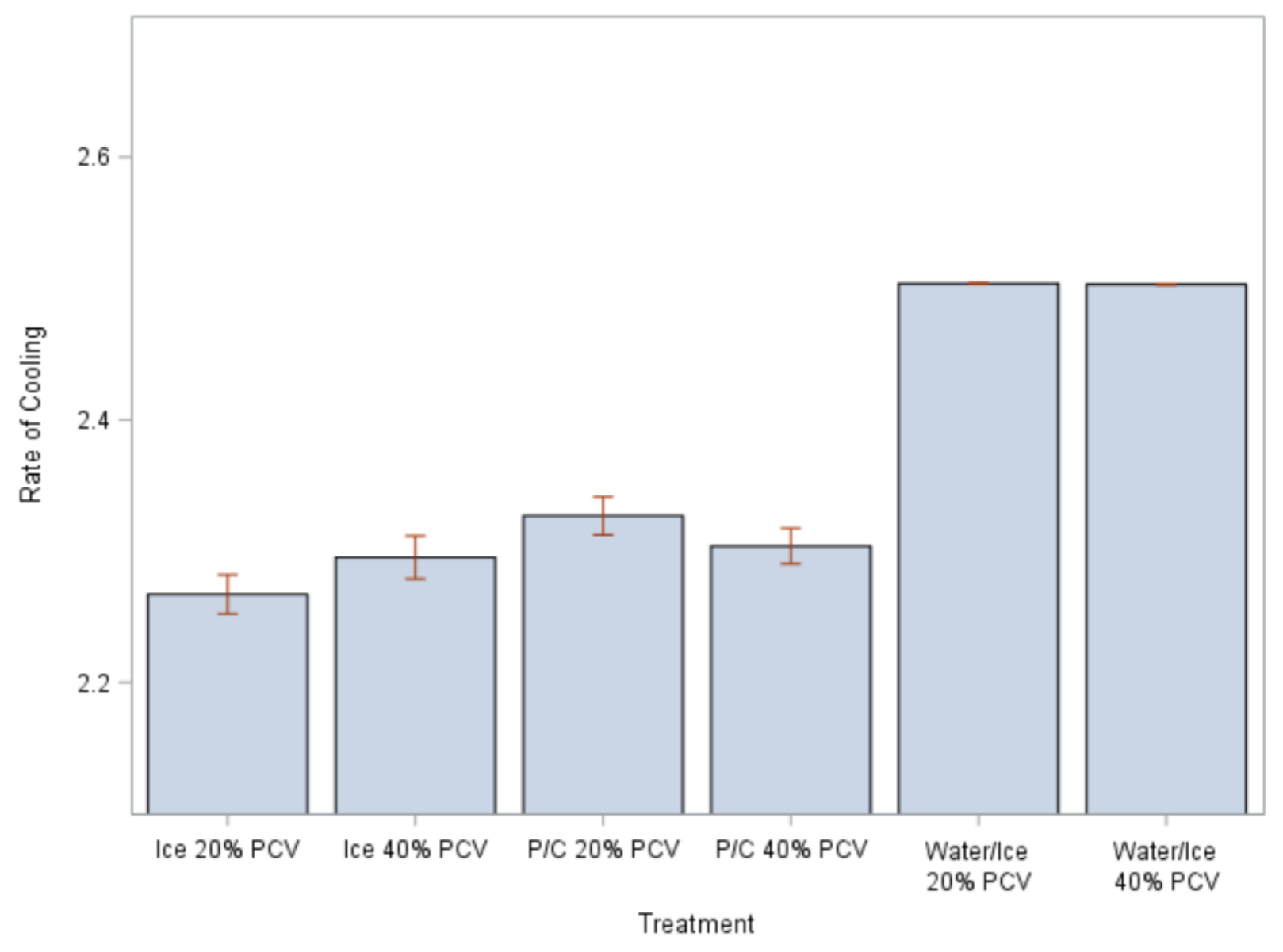

298

299

300 Ice $=$ ice bath; $\mathrm{P} / \mathrm{C}=$ precooled tube in ice bath; Water/Ice $=$ ice water bath 
